\title{
The Economic Burden of ACPA-Positive Status Among Patients with Rheumatoid Arthritis
}

\author{
Jason Shafrin, PhD; Mahlet Gizaw Tebeka, MS; Kwanza Price, MPH;
} Chad Patel, PharmD; and Kaleb Michaud, PhD

\begin{abstract}
BACKGROUND: Anticitrullinated protein antibodies (ACPAs) are serological biomarkers associated with early, rapidly progressing rheumatoid arthritis (RA), including more severe disease and joint damage. ACPA testing has become a routine tool for RA diagnosis and prognosis. Furthermore, treatment efficacy has been shown to vary by ACPA-positive status. However, it is not clear if the economic burden of patients with RA varies by ACPA status.
\end{abstract}

OBJECTIVE: To determine if the economic burden of RA varies by patient ACPA status.

METHODS: IMS PharMetrics Plus health insurance claims and electronic medical record (EMR) data from 2010-2015 were used to identify patients with incident RA. Patients were aged $\geq 18$ years, had $\geq 1$ inpatient or $\geq 2$ outpatient claims reporting an RA diagnosis code (ICD-9-CM code 714.0), and had an anticyclic citrullinated peptide (anti-CCP; a surrogate of ACPA) antibody test within 6 months of diagnosis. Incident patients were defined as those who had no claims with an RA diagnosis code in the 6 months before the first observed RA diagnosis. The primary outcome of interest was RA-related medical expenditures, defined as the sum of payer- and patient-paid amounts for all claims with an RA diagnosis code. Secondary outcomes included health care utilization metrics such as treatment with a disease-modifying antirheumatic drug (DMARD) and physician visits. Generalized linear regression models were used for each outcome, controlling for ACPA-positive status (defined as anti-CCP $\geq 20 \mathrm{AU} / \mathrm{mL}$ ), age, sex, and Charlson Comorbidity Index score as explanatory variables.

RESULTS: Of 647,171 patients diagnosed with RA, 89,296 were incident cases, and $47 \%(n=42,285)$ had an anti-CCP test. After restricting this sample to patients with a linked EMR and reported anti-CCP test result, 859 remained, with $24.7 \%(n=212)$ being ACPA-positive. Compared with ACPA-negative patients, adjusted results showed that ACPA-positive patients were more likely to use either conventional $(71.2 \%$ vs. $49.6 \%$; $P<0.001)$ or biologic $(20.3 \%$ vs. $11.8 \% ; P<0.001)$ DMARDs during the first year after diagnosis and had more physician visits (5.58 vs. 3.91 times per year; $P<0.001$ ). Annual RA-associated total expenditures were $\$ 7,941$ for ACPA-positive and $\$ 5,243$ for ACPA-negative patients $(\Delta=\$ 2,698$; $P=0.002$ ). RA-associated medical expenditures were $\$ 4,380$ for ACPApositive and $\$ 3,427$ for ACPA-negative patients $(\Delta=\$ 954 ; P=0.168)$, whereas DMARD expenditures were $\$ 3,560$ and $\$ 1,817$, respectively $(\Delta=\$ 1,743 ; P=0.001)$.

CONCLUSIONS: RA-related economic burden is higher for patients who are ACPA-positive compared with those who are ACPA-negative. Providers may wish to inform patients diagnosed with ACPA-positive RA about the likely future disease and economic burden in hopes that both stakeholders can be more proactive in addressing them.

J Manag Care Spec Pharm. 2018;24(1):4-11

Copyright $\odot 2018$, Academy of Managed Care Pharmacy. All rights reserved.

\section{What is already known about this subject}

Anticitrullinated protein antibodies (ACPAs) are established biomarkers for identifying early, rapidly progressing rheumatoid arthritis (RA).

The efficacy of RA treatments varies depending on whether patients are ACPA-positive

\section{What this study adds}

This study is the first to measure the economic burden of patients with RA by ACPA status in the United States.

RA-associated total expenditures were 51\% higher among patients with the ACPA biomarker compared with those without the biomarker.

Ј n the United States, 1.3 million people have rheumatoid arthritis (RA). RA patients incur $\$ 19.3$ billion in direct and indirect costs in the United States annually. ${ }^{1-3}$ While there is a large number of treatments available for RA, patients often try multiple therapies before finding an optimal one, and the effectiveness of some therapies declines over time. Biomarkers such as anticitrullinated protein antibodies (ACPAs) and rheumatoid factor (RF) can be used to better understand disease progression. $^{4,5}$

In particular, the ACPA serological biomarker of RA is being increasingly used to inform patient prognosis. ${ }^{6,7}$ As medicine has moved toward a more personalized treatment approach in recent years, published research has found that being ACPApositive is an independent predictor of response for some biologic disease-modifying antirheumatic drugs (bDMARDs) more than others. ${ }^{8,9}$ DMARDs act on the immune system and block inflammation to slow the progression of RA; targeted DMARDs block pathways inside immune cells whereas bDMARDs are produced by living cells and work on immune proteins called cytokines. ${ }^{10}$ There is a wide range of published estimates for the percentage of patients with RA who are ACPA-positive, ranging from a low of $5 \%$ to as high as $91 \% .{ }^{11-13}$ ACPA status has crucial prognostic value for RA diagnosis and observation of RA progression, as it can be observed in serum samples earlier than RF. ${ }^{6,14}$ The RF biomarker, on the other hand, has not shown the same predictive power. ${ }^{8,15}$ However, both ACPA and RF are associated with more severe disease and joint damage. ${ }^{6,16}$ 


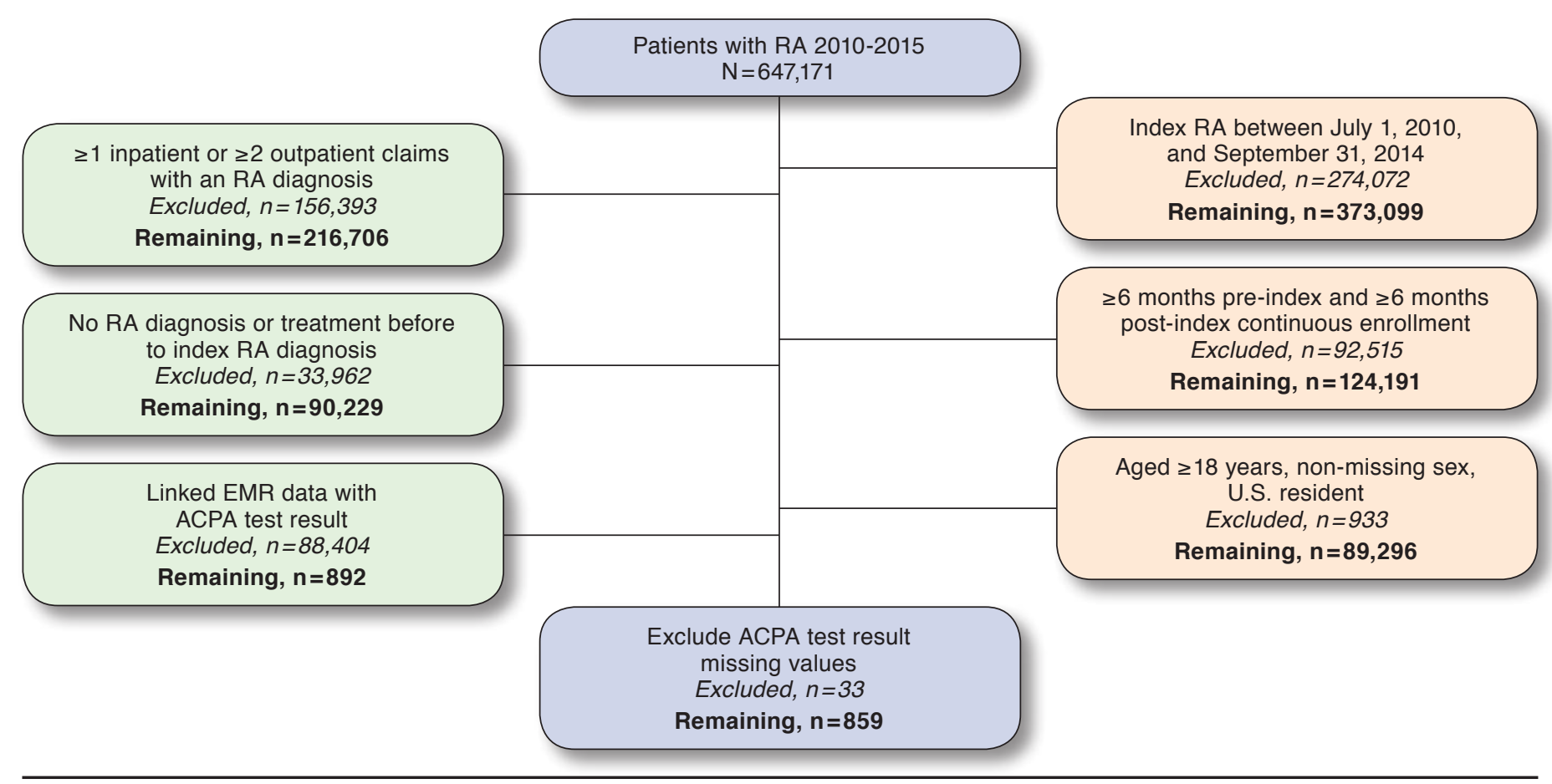

$A C P A=$ anticitrullinated protein antibody; EMR = electronic medical record; $R A=$ rheumatoid arthritis.

This is because ACPA and RF formation occurs prior to clinical manifestation of RA and irreversible joint damage. . $^{17,18}$

Despite the relatively recent understanding of the importance of ACPA status on treatment response to specific medications, ${ }^{19}$ little is known about the differences in the economic burden of disease for ACPA-positive and ACPA-negative patients. While there has been substantial research into the health care resource utilization and costs of RA in general, the lack of specific data on ACPA status may result in a misunderstanding of the uniqueness of these subpopulations in terms of burden and unmet need. Further, ACPA status may be particularly relevant for patients who have an early, rapidly advancing form of RA. ${ }^{15}$ Recent studies from phase $3 \mathrm{~b}$ clinical trials have shown benefit of some biologic DMARDs in this specific population. ${ }^{20-22}$

As understanding of the importance of ACPA status on treatment response to specific medications, particularly as a component of defining the early, rapidly progressing RA patient, is fairly recent, ${ }^{23}$ little is known about the differences in burden of disease for ACPA-positive and ACPA-negative patients. This study measured the difference in economic cost burden between patients with RA who were ACPA-positive compared with those who were ACPA-negative using a health insurance claims database linked to an electronic medical record (EMR).

\section{Methods}

\section{Study Design}

This study is an observational, retrospective cohort study that measured differences in the economic burden of RA between patients with newly diagnosed (i.e., incident) cases of RA who are ACPA-positive compared with those who are not. We used health insurance claims data linked to EMR data containing laboratory results. After conducting sensitivity analysis differentiating ACPA as strongly or weakly positive, we defined patients as ACPA-positive if their anticyclic citrullinated peptide (anti-CCP) test result was $\geq 20 \mathrm{AU} / \mathrm{mL}^{.}{ }^{24}$

\section{Data Source}

We used 5 years (2010-2015) of IMS PharMetrics Plus data for this study. The aggregated IMS PharMetrics Plus database is composed of adjudicated claims for more than 150 million unique enrollees across the United States. Enrollees with both medical and pharmacy coverage in 2013 represent 40 million active lives. The PharMetrics Plus data have diverse representation of geography, employers, payers, providers, and therapy areas. Patients in the majority of 3-digit ZIP codes and in every metropolitan statistical area of the United States are represented, with coverage of data from $90 \%$ of U.S. hospitals and $80 \%$ of all U.S. doctors and representation from $85 \%$ of the 
TABLE 1 Descriptive Statistics by ACPA Status ${ }^{a}$

\begin{tabular}{|c|c|c|c|c|}
\hline & $\begin{array}{c}\text { ACPA-Negative } \\
(\mathrm{n}=647)\end{array}$ & $\begin{array}{c}\text { ACPA-Positive } \\
(\mathrm{n}=212)\end{array}$ & $P$ Value & $\begin{array}{c}\text { Total } \\
(\mathrm{N}=859)\end{array}$ \\
\hline \multicolumn{3}{|c|}{ Age group, years n (\%) } & 0.384 & \\
\hline $18-34$ & $72(11.1)$ & $18 \quad(8.5)$ & & $90 \quad(10.5)$ \\
\hline $35-44$ & $116 \quad(17.9)$ & $31 \quad(14.6)$ & & $147 \quad(17.1)$ \\
\hline $45-54$ & $172(26.6)$ & $69 \quad(32.5)$ & & $241 \quad(28.1)$ \\
\hline $55-64$ & $210(32.5)$ & $70(33.0)$ & & $280 \quad(32.6)$ \\
\hline $65+$ & 77 (11.9) & $24 \quad(11.3)$ & & $101 \quad(11.8)$ \\
\hline Male, n (\%) & $153(23.6)$ & $66 \quad(31.1)$ & 0.030 & $219 \quad(25.4)$ \\
\hline \multicolumn{3}{|c|}{ CCI score, $\mathrm{n}(\%)$} & 0.266 & \\
\hline 0 & $442(68.3)$ & $157(74.1)$ & & $599 \quad(69.7)$ \\
\hline 1 & $126(19.5)$ & $32(15.1)$ & & $158(18.4)$ \\
\hline 2 & $55 \quad(8.5)$ & $13 \quad(6.1)$ & & $68 \quad(7.9)$ \\
\hline$\geq 3$ & $24 \quad(3.7)$ & $10 \quad(4.7)$ & & $34 \quad(4.0)$ \\
\hline \multicolumn{3}{|c|}{ Region, n (\%) } & $<0.001$ & \\
\hline East & $196(30.3)$ & $97 \quad(45.8)$ & & $293(34.1)$ \\
\hline Midwest & $81 \quad(12.5)$ & $39(18.4)$ & & $120(14.0)$ \\
\hline South & $279(43.1)$ & $60(28.3)$ & & $339 \quad(39.5)$ \\
\hline West & $91 \quad(14.1)$ & $16 \quad(7.5)$ & & $107 \quad(12.5)$ \\
\hline
\end{tabular}

a ACPA-positive $=$ anti- $C C P \geq 20 \mathrm{AU} / \mathrm{mL}$

$A C P A=$ anticitrullinated protein antibody; $C C I=$ Charlson Comorbidity Index.

Fortune 100 companies. IMS PharMetrics Plus data have been used in previous RA studies. ${ }^{25,26}$

To obtain ACPA status, the PharMetrics Plus dataset was linked to available ambulatory EMR records. The EMR data contained laboratory results that were used to measure ACPA and RF from a patient's anti-CCP and RF tests, respectively.

\section{Study Population}

Our dataset contained 647,171 distinct patients with an RA diagnosis (International Classification of Diseases, Ninth Revision, Clinical Modification [ICD-9-CM] code 714.0). We matched the RA cohort selected based on the PharMetrics Plus health plan claims database using the deidentified patient number with the IMS ambulatory EMR database. EMR-linked patients with valid ACPA test results were selected to be included in the analytical sample.

To be included in the analysis, patients were required to have $\geq 1$ inpatient or $\geq 2$ outpatient claims with an ICD-9-CM diagnosis code of 714.0, similarly to other claims-based RA analyses in the literature. ${ }^{27}$ Patients were required to have $\geq 2$ outpatient claims because these claims data may contain so-called "rule-out" diagnoses where the physician includes the diagnosis code for tests for a specific condition even when the patient does not have the condition. ${ }^{28}$ Patients were also required to have an anti-CCP test within 6 months of diagnosis, be at least 18 years of age at the time they were diagnosed, and be continuously enrolled for at least 6 months after diagnosis in a health plan covering medical and pharmacy expenditures. Patients living outside of the United States were excluded from the study population.
To identify newly diagnosed patients, we followed a previous work ${ }^{29}$ by requiring at least a 6 -month "clean period" with no RA diagnosis before the first observed RA diagnosis in the data.

\section{Key Variables of Interest}

The primary outcome of interest was health care costs. Costs were defined as payments made by both payers and patients (i.e., out-of-pocket deductibles, coinsurance, and copayments) and were captured over a 12-month period following diagnosis. Costs measured included pharmacy and medical costs (e.g., inpatient, outpatient, emergency department [ED], physician visits). Both all-cause costs and RA-related costs were analyzed, with the latter defined from all medical claims carrying an ICD-9-CM diagnosis code for RA in any diagnosis position. All costs were expressed in 2015 constant dollars, adjusted using the medical care component of the Consumer Price Index.

The secondary outcome of interest was RA-related health care resource utilization. This included hospitalizations, ED visits, physician visits, and number of prescriptions. For the small proportion of patients who had a follow-up period less than 12 months, we annualized their health care costs and utilization to reflect an annual rate.

The key independent variable of interest was whether patients were ACPA-positive. ACPA-positive status was defined by anti-CCP test $\geq 20 \mathrm{AU} / \mathrm{mL}$ per American College of Rheumatology (ACR) guidelines. ${ }^{19,30}$ Additional independent variables included patient demographics (age, gender), region of residence, and patient health status (Charlson Comorbidity Index [CCI] score).

\section{Statistical Analyses}

For each outcome variable, descriptive statistics were tabulated through univariate analysis by ACPA status. The distributions of test results were also presented as means, medians, standard deviations, and ranges. Patient characteristics including age, gender, region, and other demographic and clinical variables (e.g., comorbidities) were summarized. In addition, Wilcoxon rank-sum tests were conducted to determine whether any differences in resource use or cost were statistically significant across these cohorts.

Health care costs were measured using the financial fields on administrative claims in the IMS PharMetrics Plus databases and include the gross covered payments for all health care services or products (i.e., the amount eligible for payment after applying pricing guidelines such as fee schedules and discounts but including deductibles, copayments, and coordination of benefits amounts). All costs were expressed in 2015 constant dollars, adjusted using the medical care component of the Consumer Price Index..$^{31}$

Health care spending was compared using a regression analysis to control for observed differences between ACPA-positive and ACPA-negative patients. In our baseline approach, we used a generalized linear model (GLM) to adjust for confounders/ 


\begin{tabular}{|c|c|c|c|c|c|c|}
\hline \multirow[b]{3}{*}{ Prescription drug costs } & \multicolumn{3}{|c|}{ RA-Associated Costs ${ }^{b}$} & \multicolumn{3}{|c|}{ All-Cause Costs } \\
\hline & $\begin{array}{c}\text { ACPA-Negative } \\
\mathrm{n}=647\end{array}$ & $\begin{array}{c}\text { ACPA-Positive } \\
n=212\end{array}$ & \multirow{2}{*}{$\begin{array}{c}\text { Difference } \\
1,819^{c}\end{array}$} & $\begin{array}{l}\text { ACPA-Negative } \\
\mathrm{n}=647\end{array}$ & $\begin{array}{l}\text { ACPA-Positive } \\
\mathrm{n}=212\end{array}$ & \multirow{2}{*}{$\begin{array}{c}\text { Difference } \\
316\end{array}$} \\
\hline & $1,798 \quad(5,807)$ & $3,617 \quad(7,961)$ & & $4,780(10,287)$ & $5,096 \quad(8,621)$ & \\
\hline Medical costs & $3,423 \quad(8,677)$ & $4,390 \quad(8,768)$ & 967 & $13,662 \quad(23,148)$ & $10,790 \quad(16,497)$ & $-2,872$ \\
\hline Inpatient & $1,670 \quad(7,152)$ & $1,324 \quad(6,314)$ & -346 & $4,098 \quad(14,388)$ & $2,584 \quad(9,518)$ & $-1,514$ \\
\hline Outpatient & $1,754 \quad(4,131)$ & $3,066 \quad(6,411)$ & $1,312^{\mathrm{d}}$ & $9,565 \quad(14,849)$ & $8,206 \quad(11,418)$ & $-1,359$ \\
\hline Doctor visit & $(685)$ & $(622)$ & $209^{c}$ & $2,530 \quad(2,428)$ & $2,042 \quad(1,844)$ & $-488^{d}$ \\
\hline ED & $(882)$ & $(695)$ & -33 & $305 \quad(1,307)$ & $(851)$ & -143 \\
\hline Outpatient hospital & $1,000 \quad(3,924)$ & $2,154 \quad(6,407)$ & $1,154^{\mathrm{d}}$ & $5,278(13,364)$ & $5,185 \quad(10,419)$ & -93 \\
\hline Other outpatient & $108 \quad(460)$ & $89 \quad(295)$ & -19 & $1,451 \quad(2,651)$ & $818 \quad(1,755)$ & $-633^{d}$ \\
\hline Total costs & $5,222 \quad(10,382)$ & $8,007 \quad(11,801)$ & $2,785^{\mathrm{d}}$ & $18,442(28,366)$ & $15,886(19,088)$ & $-2,556$ \\
\hline \multicolumn{7}{|c|}{$\begin{array}{l}\text { Incident cohort }=859 \text {; all values are shown as mean (SD) in } 2015 \text { U.S. dollars. } \\
\text { bRA-associated prescription drug costs only include spending on DMARDs. } \\
{ }^{c} P<0.01 \text {. } \\
d_{P}<0.05 \text {. }\end{array}$} \\
\hline
\end{tabular}

covariates. This approach was used both for the cost and utilization numbers; since the utilization numbers are annualized, they may not be integer values and thus the same GLM was used.

\section{Sensitivity Analyses}

We conducted 2 sensitivity analyses. First, rather than measure patients who are likely rapidly progressing or have a poor prognosis using a single ACPA-positive indicator, we used the ACR definition of rapidly progressing or poor prognosis patients, which requires the presence of at least 1 of the following clinical dimensions: anti-CCP test $\geq 60 \mathrm{AU} / \mathrm{mL}$ or $\mathrm{RF}$ test $\geq 15 \mathrm{IU} / \mathrm{mL}$ or the presence of $\mathrm{RA}$ vasculitis (ICD-9-CM diagnosis code 714.2) or Felty's syndrome (ICD-9-CM diagnosis code 714.1). ${ }^{19}$ We also compared differences in cost between patients who had no serological biomarkers against those with a single biomarker (ACPA-positive or RF-positive) compared against those who were both ACPApositive and RF-positive. In our second sensitivity analysis, we no longer limited the analysis to patients with incident RA but also included patients with prevalent forms of RA. For the prevalent RA patient analysis, we no longer required a 6-month clean period prior to first diagnosis.

\section{Results}

\section{Study Population}

Of 647,171 patients diagnosed with RA, 89,296 were incident cases meeting inclusion criteria and $47 \%(n=42,285)$ had an anti-CCP test. After restricting the sample to 9,747 patients with a linked EMR, 859 reported an ACPA test result (Figure 1). Of these 859 patients with newly diagnosed RA, 24.7\% were ACPA-positive.
The ages of individuals in the study ranged from 18-84 years at index RA diagnosis. About 3 out of every 5 patients with RA were aged between 45 and 64 years, and 12\% were 65 years or older. Three quarters of RA patients were female, similar to the gender composition nationally of patients with RA. ${ }^{32}$ Age and gender distributions were similar comparing ACPA-positive and ACPA-negative patients (Table 1).

\section{Health Care Costs}

Health care costs directly associated with the treatment of patients with RA were higher among ACPA patients, and all-cause costs were similar across ACPA status. Descriptive analysis showed no statistically significant difference in allcause health care cost by ACPA status (Table 2). Results showed that overall expenditures for ACPA-positive patients were higher compared to ACPA-negative patients. On the other hand, ACPA-positive patients had significantly higher RA-associated prescription costs $(P<0.01)$, outpatient service costs $(P<0.05)$, and total costs $(P<0.05)$ compared with ACPAnegative patients. However, the differences were less significant and even reversed for all-cause costs. ACPA-positive patients had significantly lower all-cause expenditures for doctor visits and other outpatient services (i.e., all outpatient costs excluding costs associated with physician visits, ED visits, and outpatient hospital claims; $P<0.01$ ).

Similar differences were observed in multivariable analyses controlling for differences in patient characteristics. After controlling for age, gender, and CCI score in the multivariate regression analysis (Table 3), all-cause cost was $\$ 2,430$ $(P=0.236)$ lower among ACPA-positive individuals, with higher prescription drug cost $(\Delta=+\$ 294, P=0.707)$ and lower medical cost $(\Delta=-\$ 2,725, P=0.107)$ among ACPA-positive compared 
The Economic Burden of ACPA-Positive Status Among Patients with Rheumatoid Arthritis

TABLE 3 Multivariable Analysis of Health Care Costs by ACPA Status, Baseline Analysis Using Incident Cohort $(\mathrm{N}=859)^{\mathrm{a}}$

\begin{tabular}{|c|c|c|c|c|c|c|}
\hline & \multicolumn{3}{|c|}{ ACPA-Positive vs. ACPA-Negative } & \multicolumn{3}{|c|}{ Adjusted Mean (SE) } \\
\hline & Difference & $95 \%$ CI & $P$ Value & ACPA-Positive & ACPA-1 & Tegative \\
\hline \multicolumn{7}{|l|}{ RA-associated costs } \\
\hline Total & 2,698 & 1,025 to 4,370 & 0.002 & $7,941 \quad(740)$ & 5,243 & $(423)$ \\
\hline Prescription drug & 1,743 & 753 to 2,734 & 0.001 & $3,560 \quad(438)$ & 1,817 & $(251)$ \\
\hline Medical costs & 953 & -402 to 2,309 & 0.168 & $4,380 \quad(600)$ & 3,427 & $(343)$ \\
\hline Inpatient & -388 & $-1,471$ to 694 & 0.482 & $1,292 \quad(479)$ & 1,680 & $(274)$ \\
\hline Outpatient & 1,343 & 596 to 2,089 & $<0.001$ & $3,089 \quad(330)$ & 1,746 & $(189)$ \\
\hline Doctor visit & 215 & 110 to 319 & $<0.001$ & $(46)$ & 543 & $(26)$ \\
\hline ED visit & -28 & -159 to 103 & 0.675 & $(58)$ & 99 & (33) \\
\hline Outpatient hospital & 1,175 & 449 to 1,901 & 0.002 & $2,170 \quad(321)$ & 995 & $(184)$ \\
\hline Other outpatient & -19 & -85 to 47 & 0.565 & $(29)$ & 108 & $(17)$ \\
\hline \multicolumn{7}{|l|}{ All-cause costs } \\
\hline Grand total & $-2,430$ & $-6,453$ to 1,592 & 0.236 & $15,981 \quad(1,780)$ & 18,411 & $(1,017)$ \\
\hline Prescription drug & 294 & $-1,240$ to 1,828 & 0.707 & $(679)$ & 4,785 & (388) \\
\hline Medical costs & $-2,725$ & $-6,033$ to 585 & 0.107 & $10,901 \quad(1,464)$ & 13,626 & $(837)$ \\
\hline Inpatient & $-1,467$ & $-3,520$ to 586 & 0.161 & $2,619 \quad(908)$ & 4,086 & $(519)$ \\
\hline Outpatient & $-1,257$ & $-3,422$ to 909 & 0.255 & $8,283 \quad(958)$ & 9,540 & $(548)$ \\
\hline Doctor visit & -448 & -803 to -94 & 0.013 & $2,072 \quad(157)$ & 2,520 & (90) \\
\hline ED visit & -134 & -322 to 55 & 0.164 & $(83)$ & 303 & $(48)$ \\
\hline Outpatient hospital & -39 & $-2,001$ to 1,923 & 0.969 & $5,226 \quad(868)$ & 5,265 & $(496)$ \\
\hline Other outpatient & -635 & $-1,014$ to -257 & 0.001 & (168) & 1,451 & (96) \\
\hline
\end{tabular}

${ }^{a}$ Costs are given in 2015 U.S. dollars.

$A C P A=$ anticitrullinated protein antibody; $C I=$ confidence interval; $E D=$ emergency department; $R A=$ rheumatoid arthritis; $S E=$ standard error

with ACPA-negative individuals. None of these results, however, were statistically different from zero.

On the other hand, annual RA-associated total expenditures were $\$ 7,941$ for ACPA-positive and $\$ 5,243$ for ACPA-negative patients $(\Delta=\$ 2,698, P=0.002$; Figure 2). This difference represents a $51 \%$ increase in overall RA-associated costs. RA-associated medical expenditures (excluding prescription drug costs) were $\$ 4,380$ for ACPA-positive and $\$ 3,427$ for ACPA-negative patients $(\Delta=\$ 953 ; P=0.168)$.

\section{Health Care Resource Utilization}

Descriptive analysis of health care resource utilization also showed higher RA-associated health care utilization among ACPA-positive patients compared with ACPA-negative patients (Appendix A, available in online article). Specifically, ACPApositive patients were more likely to use either conventional (71.2\% vs. $49.6 \% ; P<0.001)$ or biologic $(20.3 \%$ vs. $11.8 \%$; $P<0.001)$ DMARDs during the first year after diagnosis and had more physician visits (5.58 vs. 3.91 times per year; $P<0.001$ ).

\section{Sensitivity Analyses}

The results were similar among the rapidly progressing subgroup; ACPA-positive patients had higher costs than ACPA-negative patients (Appendix B, available in online article). Compared with patients without serological biomarkers (i.e., neither ACPA-positive nor RF-positive), patients with 1 (but not both) biomarkers had similar RA-related total cost $(\Delta=\$ 701 ; P=0.477)$, but patients who were ACPA-positive and RF-positive had significantly higher RA-related total cost $(\Delta=\$ 2,748 ; P=0.021)$. In addition, the overall pattern of disease burden by ACPA status in the prevalent cohort $(\mathrm{N}=2,524)$ was similar to the findings from the primary analysis in the incident cohort. However, differences in cost by ACPA status are less apparent, potentially due to the more heterogeneous nature of the prevalent cohort, which contains a mix of patients who have varying disease prognosis and severity or unobserved differences in disease severity across ACPA-positive and ACPA-negative patients.

\section{Discussion}

ACPA-positive patients generally had higher RA-related costs. Annual RA-associated expenditures were \$2,698 higher for ACPA-positive compared with ACPA-negative patients $(P=0.002)$. Setting aside the cost for DMARDs, annual medical cost was $\$ 954$ higher for ACPA-positive compared with ACPAnegative patients $(P=0.168)$. The difference in medical cost by ACPA status was mainly driven by outpatient hospital costs $(\$ 1,175$ higher; $P=0.002)$ and doctor visit costs ( $\$ 214$ higher; $P<0.001$ ). Similar patterns were observed in sensitivity analyses conducted using rapidly progressing status and a prevalent 


\section{FIGURE 2 Baseline Results: Annual RA-Associated Health Care Costs by ACPA Status in} Incident Cohort ( $\mathrm{N}=859$ )

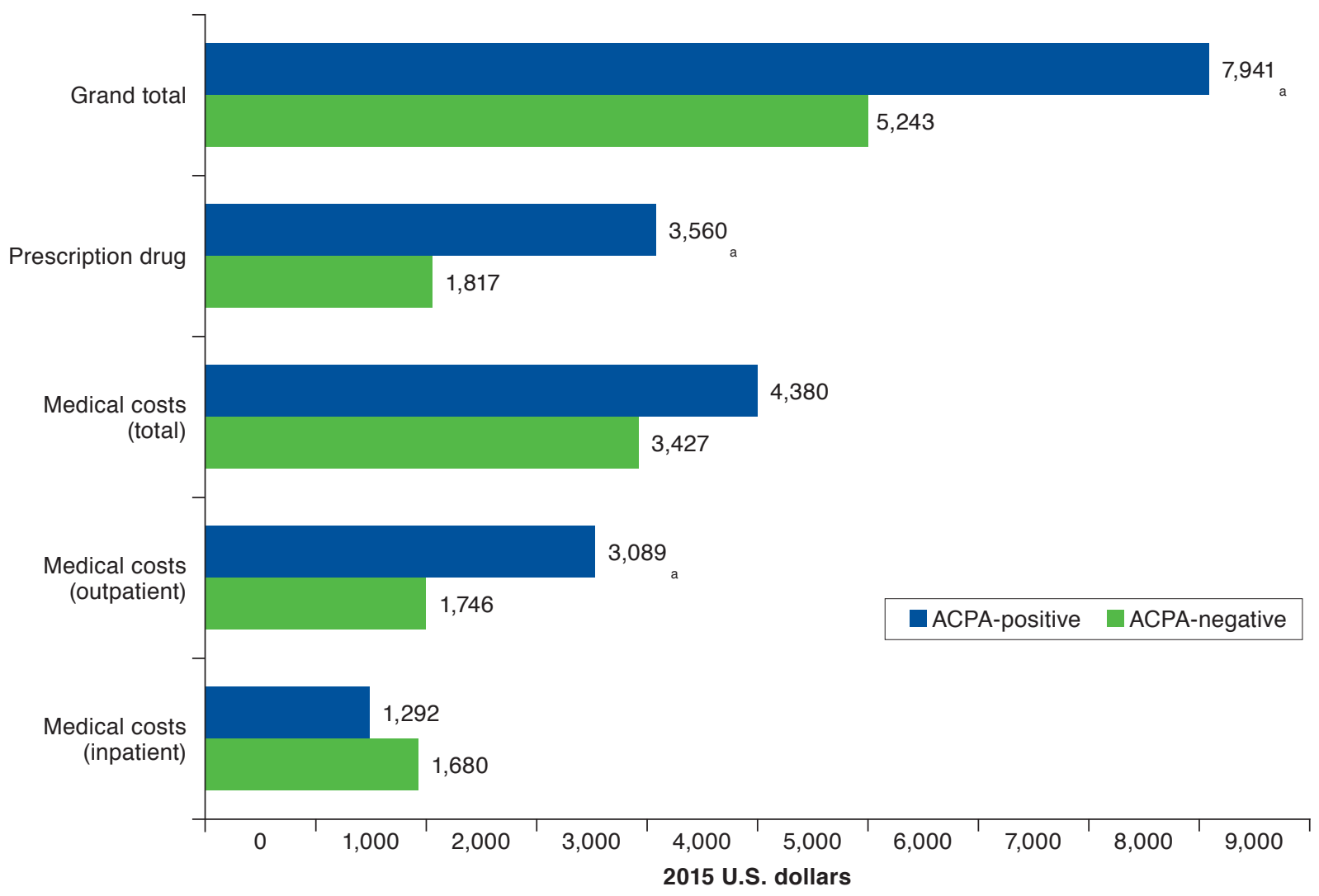

${ }^{a} P<0.05$.

$A C P A=$ anticitrullinated protein antibody; $R A=$ rheumatoid arthritis.

cohort. On the other hand, we found no statistically significant difference in overall cost between patients with RA who were ACPA-positive compared with ACPA-negative.

Overall, the results suggest that ACPA-positive patients may have more aggressive treatment of RA and higher RA-related disease burden but also may be in better overall health due to a decreased prevalence of comorbid conditions. Although speculative, the observed difference in RA-related cost aligns with results from the literature that ACPA positivity is associated with more severe disease progression and joint damage. ${ }^{6,16}$ At the same time, the failure to find any difference in overall cost between ACPA-positive and ACPA-negative patients could be interpreted to mean that there is truly no difference in spending across these groups or that overall disease burden outside of RA or RA disease progression itself may vary across patients by ACPA status. For instance, whereas $74.1 \%$ of ACPA-positive patients had a CCI score of 0 , for ACPA-negative patients, this figure was $68.3 \%$. More broadly, this study's estimates of the economic burden of being ACPA-positive align with the findings from a study of the economic burden of patients with RA in Italy. ${ }^{33}$

Research from other studies has provided some suggestions of clinical approaches to improve patient health outcomes among ACPA-positive patients, although the effect of these treatments on economic burden is uncertain. The ACR suggests the use of DMARDs and biologic agents or biologics based on indications of early RA followed by established RA. ${ }^{19}$ Although many of the biologic agents are expensive relative to conventional DMARDs, recent research suggests, however, that certain bDMARDs may be more efficacious among ACPA-positive patients compared with ACPA-negative patients compared with the more widely used tumor necrosis factor inhibitor class. ${ }^{23}$ Given the various options for treatment, the results for this study imply that evidence on ACPA status can be important in the choice of treatment. 


\section{Limitations}

This study has a number of limitations. First, not all patients or even all patients with RA receive regular anti-CCP or RF tests, and it is likely that these lab tests were not applied randomly. Although our baseline approach is limited to patients receiving an anti-CCP test, our sensitivity analysis also examines the economic burden among RA patients based on whether they are ACPA-positive or RF-positive.

Second, although our health insurance claims data captured any patient out-of-pocket payments from services covered in part by insurers, we could not observe any patient health care spending made without knowledge of the payer. Furthermore, we allowed for ICD-9-CM codes to count as valid diagnoses if they were listed in any diagnosis position on the claim, which may be a less specific indicator of RA status than would be the case of requiring a primary diagnosis code. ${ }^{34}$

Third, the sample size of our analysis is fairly small, less than 1,000 people. To address this issue, we conducted a sensitivity analysis to expand our comparison to patients with prevalent RA and we found qualitatively similar results. Fourth, the sample comes from a largely commercially insured patient population, and individuals aged 65 years and above are underrepresented. This challenge has been faced by other studies using administrative datasets, ${ }^{35,36}$ and researchers should be cautious in extrapolating the results of this study beyond the commercially insured population. Fifth, the EMR data for this study only included the lab results; thus, we were unable to include disease severity measures to assess baseline disease states for the ACPA subpopulations. In other words, the demographics and comorbidities captured in this study do not fully capture disease severity. However, DMARD use is much higher among ACPA-positive patients in the study sample, suggesting higher disease severity.

Finally, the results of this study should be interpreted as demonstrating the correlation between ACPA status and economic burden as there exists significant uncertainty regarding the direction of causation of ACPA status on economic burden. On one hand, it could be the case that physicians believe that these patients who are ACPA-positive will have higher RA-related disease burden and thus they would be more likely to prescribe more aggressive medications and request more frequent followup visits. On the other hand, physicians may treat ACPA-positive patients similarly to ACPA-negative patients, but ACPApositive patients may be more likely to have RA-related complications due to more rapidly progressing disease.

\section{Conclusions}

This study found that patients who were ACPA-positive had a similar overall cost burden but had higher RA-related costs compared with patients who were ACPA-negative. Future research should examine the relationship between ACPA status, disease severity, and economic outcomes and should also examine whether targeting RA-related treatment based on clinical factors such as ACPA-positive status leads to differential clinical and economic outcomes.

\section{Authors}

JASON SHAFRIN, PhD, and MAHLET GIZAW TEBEKA, MS, Precision Health Economics, Los Angeles, California. KWANZA PRICE, MPH, and CHAD PATEL, PharmD, Bristol-Myers Squibb, Princeton Pike, New Jersey. KALEB MICHAUD, PhD, University of Nebraska Medical Center, Omaha, and National Data Bank for Rheumatic Diseases, Wichita, Kansas.

AUTHOR CORRESPONDENCE: Jason Shafrin, PhD, Precision Health Economics, 11100 Santa Monica Blvd., Ste. 500, Los Angeles, CA 90025. Tel.: 310.984.7705; E-mail: jason.shafrin@precisionhealtheconomics.com.

\section{DISCLOSURES}

Funding for this research was contributed by Bristol-Myers Squibb. Patel and Price are employees and stockholders of Bristol-Myers Squibb. Shafrin and Tebeka are employees of Precision Health Economics, a health care consulting firm that received funding from Bristol-Myers Squibb to conduct this study. Michaud has received a grant from Pfizer and is employed by the National Data Bank for Rheumatic Diseases, which has received funds from Amgen, Bristol-Myers Squibb, Eli Lilly, Janssen, Pfizer, and Regeneron.

Study concept and design were contributed by Shafrin, Price, Patel, and Michaud. Shafrin, Price, and Patel collected the data, and all authors contributed equally to data analysis. The manuscript was written by Shafrin and Tebeka and revised by Shafrin, Price, Patel, and Michaud.

\section{REFERENCES}

1. Birnbaum H, Pike C, Kaufman R, Marynchenko M, Kidolezi Y, Cifaldi M. Societal cost of rheumatoid arthritis patients in the U.S. Curr Med Res Opin. 2010;26(1):77-90

2. Helmick CG, Felson DT, Lawrence RC, et al. Estimates of the prevalence of arthritis and other rheumatic conditions in the United States. Part I. Arthritis Rheum. 2008;58(1):15-25

3. Kawatkar AA, Jacobsen SJ, Levy GD, Medhekar SS, Venkatasubramaniam KV, Herrinton LJ. Direct medical expenditure associated with rheumatoid arthritis in a nationally representative sample from the Medical Expenditure Panel Survey. Arthritis Care Res. 2012;64(11):1649-56.

4. Farid SSh, Azizi G, Mirshafiey A. Anti-citrullinated protein antibodies and their clinical utility in rheumatoid arthritis. Int J Rheum Dis. 2013;16(4):379-86.

5. van der Woude D, Syversen SW, van der Voort EI, et al. The ACPA isotype profile reflects long-term radiographic progression in rheumatoid arthritis. Ann Rheum Dis. 2010;69(6):1110-16.

6. Szodoray P, Szabó Z, Kapitány A, et al. Anti-citrullinated protein/peptide autoantibodies in association with genetic and environmental factors as indicators of disease outcome in rheumatoid arthritis. Autoimmun Rev. 2010;9(3):140-43

7. Smolen JS, Landewe R, Bijlsma J, et al. EULAR recommendations for the management of rheumatoid arthritis with synthetic and biological disease-modifying antirheumatic drugs: 2016 update. Ann Rheum Dis. 2017;76(6):960-77. 
8. Gottenberg JE, Ravaud P, Cantagrel A, et al. Positivity for anti-cyclic citrullinated peptide is associated with a better response to abatacept: data from the 'Orencia and Rheumatoid Arthritis' registry. Ann Rheum Dis. 2012;71(11):1815-19.

9. Pieper J, Herrath J, Raghavan S, Muhammad K, Vollenhoven R, Malmstrom V. CTLA4-Ig (abatacept) therapy modulates T cell effector functions in autoantibody-positive rheumatoid arthritis patients. BMC Immunol. 2013;14:34.

10. Arthritis Foundation. DMARDs overview. Available at: http://www. arthritis.org/living-with-arthritis/treatments/medication/drug-types/diseasemodifying-drugs/drug-guide-dmards.php. Accessed June 23, 2017.

11. Young BJ, Mallya RK, Leslie RD, Clark CJ, Hamblin TJ. Anti-keratin antibodies in rheumatoid arthritis. Br Med J. 1979;2(6182):97-99.

12. Ioan-Facsinay A, Willemze A, Robinson DB, et al. Marked differences in fine specificity and isotype usage of the anti-citrullinated protein antibody in health and disease. Arthritis Rheum. 2008;58(10):3000-08

13. Renz H. Autoimmune Diagnostics. Berlin: Walter de Gruyter GmbH; 2012.

14. Willemze A, Trouw LA, Toes RE, Huizinga TW. The influence of ACPA status and characteristics on the course of RA. Nature Rev Rheum. 2012;8(3):144-52

15. Maneiro RJ, Salgado E, Carmona L, Gomez-Reino JJ. Rheumatoid factor as predictor of response to abatacept, rituximab and tocilizumab in rheumatoid arthritis: systematic review and meta-analysis. Semin Arthritis Rheum 2013;43(1):9-17.

16. Syversen SW, Goll GL, van der Heijde D, et al. Prediction of radiographic progression in rheumatoid arthritis and the role of antibodies against mutated citrullinated vimentin: results from a 10-year prospective study. Ann Rheum Dis. 2010;69(2):345-51.

17. Schellekens GA, de Jong BA, van den Hoogen FH, van de Putte LB, van Venrooij WJ. Citrulline is an essential constituent of antigenic determinants recognized by rheumatoid arthritis-specific autoantibodies. J Clin Invest. 1998;101(1):273-81.

18. van Venrooij WJ, van Beers JJ, Pruijn GJ. Anti-CCP antibodies: the past, the present and the future. Nat Rev Rheumatol. 2011;7(7):391-98.

19. Singh JA, Furst DE, Bharat A, et al. 2012 update of the 2008 American College of Rheumatology recommendations for the use of disease-modifying antirheumatic drugs and biologic agents in the treatment of rheumatoid arthritis. Arthritis Care Res. 2012;64(5):625-39.

20. Bristol-Myers Squibb. Analyses from phase IIIb study provide additional data in earlier use of Orencia (abatacept) plus methotrexate (MTX) in citrullinated protein (CCP)-positive adult patients with early rheumatoid arthritis (RA). Press release. November 17, 2014. Available at: https://news.bms.com/ press-release/rd-news/analyses-phase-iiib-study-provide-additional-dataearlier-use-orencia-abatacep. Accessed June 23, 2017.

21. Emery P, Burmester GR, Bykerk VP, et al. Evaluating drug-free remission with abatacept in early rheumatoid arthritis: results from the phase $3 \mathrm{~b}$, multicentre, randomised, active-controlled AVERT study of 24 months, with a 12-month, double-blind treatment period. Ann Rheum Dis. 2015;74(1):19-26.
22. Westhovens R, Robles M, Ximenes AC, et al. Clinical efficacy and safety of abatacept in methotrexate-naive patients with early rheumatoid arthritis and poor prognostic factors. Ann Rheum Dis. 2009;68(12):1870-77.

23. Sokolove J, Schiff M, Fleischmann R, et al. Impact of baseline anti-cyclic citrullinated peptide- 2 antibody concentration on efficacy outcomes following treatment with subcutaneous abatacept or adalimumab: 2-year results from the AMPLE trial. Ann Rheum Dis. 2016;75(4):709-14.

24. Quest Diagnostics. Cyclic citrullinated peptide (CCP) antibody (IgG). Test Center. 2016. Available at: http://www.questdiagnostics.com/testcenter/ TestDetail.action?ntc=11173. Accessed June 23, 2017.

25. Alemao EG, Guo Z, Burns L. Testing for anti-citrullinated peptide antibodies in US clinical practice settings in patients newly diagnosed with RA-data from three databases (2007-2014). Ann Rheum Dis. 2016;75 (Suppl 2):AB0346 [Abstract]. Available at: http://ard.bmj.com/content/75/ Suppl_2/1020.2\#block-system-main. Accessed June 23, 2017.

26. Dasa V, Sun K, Scott A, Lim S. Clinical and cost outcomes from different hyaluronic acid treatments in patients with knee osteoarthritis: evidence from a US health plan claims database. Drugs Context. 2016;5:212296.

27. Gadalla SM, Amr S, Langenberg P, et al. Breast cancer risk in elderly women with systemic autoimmune rheumatic diseases: a population-based case-control study. Br J Cancer. 2009;100(5):817-21

28. Tanihara S, Okamoto E, Une H. A statistical analysis of "rule-out" diagnoses in outpatient health insurance claims in Japan. J Eval Clin Pract. 2011;17(6):1070-74.

29. Beukelman T, Xie F, Baddley JW, et al. The risk of hospitalized infection following initiation of biologic agents versus methotrexate in the treatment of juvenile idiopathic arthritis. Arthritis Res Ther. 2016;18(1):210.

30. Miriovsky BJ, Michaud K, Thiele GM, et al. Anti-CCP antibody and rheumatoid factor concentrations predict greater disease activity in men with rheumatoid arthritis. Ann Rheum Dis. 2010;69(7):1292-97.

31. Bureau of Labor Statistics. Consumer Price Index, medical care. June 23, 2016. Available at: https://www.bls.gov/cpi/data.htm. Accessed July 5, 2017.

32. Rheumatoid Arthritis Support Network. Rheumatoid arthritis (RA) 2016. Available at: https://www.rheumatoidarthritis.org/ra/. Accessed June 23, 2017.

33. Mennini FS, Marcellusi A, Gitto L, Iannone F. Economic burden of rheumatoid arthritis in Italy: possible consequences on anti-citrullinated protein antibody-positive patients. Clin Drug Investig. 2017;37(4):375-86.

34. Greenlee R, Drahos J, VanWormer J, Landgren O, Koshiol J. PS2-22: accuracy of diagnostic codes to identify rheumatoid arthritis in archived electronic health system data: support for future cancer research network studies of lymphoma risk pathways. Clin Med Res. 2013;11(3):154.

35. Carter CT, Waters HC, Smith DB. Effect of a continuous measure of adherence with infliximab maintenance treatment on inpatient outcomes in Crohn's disease. Patient Prefer Adherence. 2012;6:417-26.

36. Goutsou M, Uluscu O, Kwasnik AA, Stianchi R, Peloso PM, Cannon G. Biologics utilization for rheumatoid arthritis in the United States: an observational longitudinal study. J Med Marketing. 2013;13(2):74-81. 
The following 2 appendices provide additional details on our baseline analysis and the results from 2 sensitivity analyses. The first analysis (Appendix A) contains results from the analysis of differences in health care utilization by ACPA status. Outcomes of interest include the number of prescriptions filled, inpatient admissions, ED visits, and physician visits. Next, the results of 2 sensitivity analyses are presented (Appendix B). Appendix A compares patients with the ACR's definition of patients with early and rapidly progressing RA and compares costs to patients without this designation. Note that being ACPA-positive would qualify a person as being with early and rapidly progressing RA under the ACR definition. Appendix B examines a cohort of patients with prevalent cases of RA; our baseline analysis, on the other hand, only examines cases with incident RA.

\begin{tabular}{|c|c|c|c|c|c|c|}
\hline \multirow[t]{3}{*}{ APPENDIX A } & \multicolumn{6}{|c|}{$\begin{array}{l}\text { Descriptive Analysis of Health Care Utilization by ACPA Status, Baseline Analysis Using } \\
\text { Incident Cohort }(\mathrm{N}=859)^{a}\end{array}$} \\
\hline & \multicolumn{3}{|c|}{ RA-Associated Utilization } & \multicolumn{3}{|c|}{ All-Cause Utilization } \\
\hline & $\begin{array}{c}\begin{array}{c}\text { ACPA-Negative } \\
\mathrm{n}=647\end{array}\end{array}$ & $\begin{array}{l}\begin{array}{c}\text { ACPA-Positive } \\
\mathrm{n}=212\end{array}\end{array}$ & Difference & $\begin{array}{c}\begin{array}{c}\text { ACPA-Negative } \\
\mathbf{n}=647\end{array}\end{array}$ & $\begin{array}{l}\text { ACPA-Positive } \\
\mathrm{n}=212\end{array}$ & Difference \\
\hline Prescriptions & $4.50(6.49)$ & $7.70(7.00)$ & $3.20^{b}$ & $38.30(34.00)$ & $36.50(28.20)$ & -1.80 \\
\hline Hospital stays & $0.12(0.41)$ & $0.11 \quad(0.38)$ & -0.01 & $0.41 \quad(1.17)$ & $0.18 \quad(0.57)$ & -0.23 \\
\hline ED visits & $0.06(0.37)$ & $0.03(0.19)$ & -0.03 & $16.90(13.90)$ & $14.30(11.20)$ & $-2.60^{c}$ \\
\hline Doctor visits & $3.90(4.20)$ & $5.60(4.20)$ & $1.70^{\mathrm{b}}$ & $38.30(34.00)$ & $36.50(28.20)$ & $-1.80^{c}$ \\
\hline $\begin{array}{l}\text { a All values are shown as } \\
{ }^{b} P<0.01 \text {. } \\
\text { c } P<0.05 \text {. } \\
\text { ACPA = anticitrullinated }\end{array}$ & antibody; ED & y department, & . & 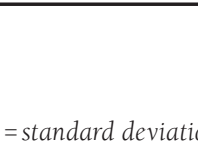 & & \\
\hline
\end{tabular}

\section{APPENDIX B Summary of Baseline and Sensitivity Analyses of RA-Associated Health Care Costs}

\begin{tabular}{|c|c|c|c|c|c|c|}
\hline \multirow{4}{*}{$\begin{array}{l}\text { Estimated Marginal } \\
\text { Effect (SE) } \\
\text { Total cost }\end{array}$} & \multirow{2}{*}{\multicolumn{2}{|c|}{$\begin{array}{c}\text { Incident Cohort } \\
\text { Baseline }(\mathrm{n}=859)\end{array}$}} & \multirow{2}{*}{\multicolumn{2}{|c|}{ Sensitivity I $(n=859)$}} & \multirow{2}{*}{\multicolumn{2}{|c|}{$\begin{array}{c}\text { Prevalent Cohort } \\
\text { Sensitivity II }(n=2,524)\end{array}$}} \\
\hline & & & & & & \\
\hline & $\begin{array}{l}\text { ACPA-Negative } \\
(\mathrm{n}=647)\end{array}$ & $\begin{array}{l}\text { ACPA-Positive } \\
\quad(n=212)\end{array}$ & $\begin{array}{c}\text { RP-Negative } \\
(\mathrm{n}=647)\end{array}$ & $\begin{array}{l}\text { RP-Positive } \\
(\mathrm{n}=212)\end{array}$ & $\begin{array}{c}\text { ACPA-Negative } \\
(\mathrm{n}=1,917)\end{array}$ & $\begin{array}{c}\text { ACPA-Positive } \\
(\mathrm{n}=607)\end{array}$ \\
\hline & $5,222 \quad(423)$ & $8,007 \quad(738)$ & $5,367 \quad(474)$ & $6,737 \quad(585)$ & $9,259 \quad(378)$ & $10,299 \quad(672)$ \\
\hline Prescription drug cost & $1,798 \quad(250)$ & $3,617 \quad(437)$ & $1,988 \quad(280)$ & $2,642 \quad(347)$ & $4,643 \quad(226)$ & $5,544 \quad(402)$ \\
\hline Total medical cost & $3,423 \quad(342)$ & $4,390 \quad(598)$ & $3,378 \quad(382)$ & $4,095 \quad(472)$ & $4,615 \quad(295)$ & $4,755 \quad(524)$ \\
\hline
\end{tabular}

